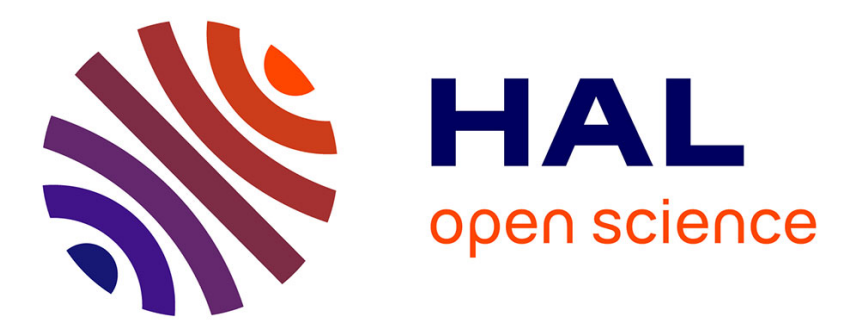

\title{
Vers une localisation syntaxique des modes verbaux Cas de la phrase indépendante
}

\author{
Lotfi Abouda
}

\section{To cite this version:}

Lotfi Abouda. Vers une localisation syntaxique des modes verbaux Cas de la phrase indépendante. Cahiers Chronos, 1998, Variations sur la référence verbale, 3, pp.293-322. halshs-01471199

\section{HAL Id: halshs-01471199 \\ https://shs.hal.science/halshs-01471199}

Submitted on 19 Feb 2017

HAL is a multi-disciplinary open access archive for the deposit and dissemination of scientific research documents, whether they are published or not. The documents may come from teaching and research institutions in France or abroad, or from public or private research centers.
L'archive ouverte pluridisciplinaire HAL, est destinée au dépôt et à la diffusion de documents scientifiques de niveau recherche, publiés ou non, émanant des établissements d'enseignement et de recherche français ou étrangers, des laboratoires publics ou privés. 


\title{
Vers une localisation syntaxique des modes verbaux Cas de la phrase indépendante ${ }^{1}$
}

\author{
Lotfi ABOUDA \\ Ecole Doctorale / UFRL - PARIS VII
}

\section{Introduction}

Partant de l'insuffisance des définitions de type logico-sémantique - qui ne sont pas de nature à emporter une adhésion unanime - et de l'absence en français d'un support morphologique systématique aux modes verbaux, nous nous proposons dans cette étude l'examen d'un certain nombre de critères syntaxiques qui vont jouer un rôle décisif dans l'apparition des différents modes. Les phénomènes syntaxiques observés (c'est-à-dire, principalement, l'élément occupant la position de Complémenteur, le type de sujet (sujet phonologiquement vide, clitique ou $N P$ lexical) ainsi que la place linéaire qu'il occupe) ne sont pas nouveaux, mais leur comparaison systématique et l'observation minutieuse de leur incidence sur l'apparition des différents modes devraient nous permettre d'avancer des généralisations descriptives intéressantes quant au rapport entre structure syntaxique et mode verbal, rendant ainsi possible une localisation syntaxique des modes verbaux et ouvrant par la même la voie à une explication sémantique.

La localisation syntaxique des modes verbaux n'est pas une hypothèse totalement originale puisqu'on la trouve déjà chez un certain nombre de grammairiens, notamment à propos du subjonctif. La nouveauté de notre hypothèse n'est donc pas l'établissement de ce lien entre syntaxe et modes, mais sa généralisation à tous les modes verbaux, y compris l'indicatif ${ }^{2}$. Se situant à

1 Ce travail a bénéficié, lors de sa présentation au $\mathrm{I}^{\mathrm{er}}$ colloque Chronos (Dunkerque, 17-18 novembre 1995), d'un certain nombre de remarques intéressantes dont nous avons essayé de rendre compte dans cette version. Nous tenons à remercier tous ceux qui nous ont aidé à en préciser les contenus linguistique et technique, notamment $\mathrm{H}$. Huot (Paris 7), A. Boushaba (ATALA), ainsi que notre ami L. Tavernier.

2 Nous refusons ainsi la hiérarchisation instaurée par la grammaire traditionnelle, et reprise plus ou moins explicitement par la plupart des linguistes, selon laquelle l'IND serait le mode zéro. " L'erreur qu'ont commise beaucoup de linguistes [...], notait Bonnard (1974: 5), semble être de tenir l'indicatif pour neutre, en donnant comme raison la plus grande fréquence de ses emplois, et le subjonctif pour chargé de valeur, parce qu'il serait la forme "marquée". En 
contre-courant des recherches menées actuellement dans le cadre de la Théorie du Gouvernement \& Liage (désormais $G B$ ), nous supposons que la localisation syntaxique des morphèmes flexionnels n'est valable que pour la catégorie modale (et personnelle), à l'exclusion de la catégorie temporelle (et probablement aspectuelle), qui, elle, obéirait à des contraintes autres que syntaxiques. Notre objectif sera ainsi de démontrer les deux hypothèses complémentaires suivantes:

(i) les modes, tous les modes, ont un lien étroit avec les caractéristiques syntaxiques de la proposition dans laquelle ils entrent. Ces caractéristiques les rendent syntaxiquement visibles;

(ii) aucune caractéristique proprement syntaxique ne peut influencer le choix d'un temps à l'exclusion des autres formes temporelles du mode considéré. On dira que la catégorie du temps est invisible pour la syntaxe.

Si ces hypothèses de départ sont correctes, il serait légitime, et même nécessaire, de distinguer entre deux types d'inacceptabilité:

(i) une inacceptabilité syntaxique (i.e. agrammaticalité), qui sera rendue ici, comme usuellement, grâce à l'astérisque $\left({ }^{*}\right)$, et qui rend compte, entre autres, des énoncés dont le mode est inacceptable, comme en ( $1 \mathrm{~b}, \mathrm{c}, \mathrm{d})$ :

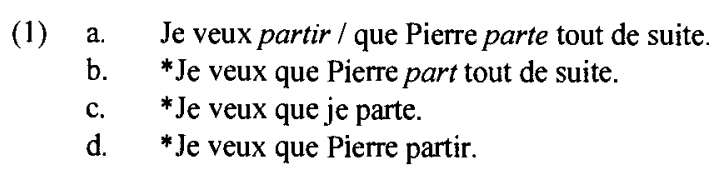

(ii) une agrammaticalité temporelle, ou plutôt une inadéquation logico-sémantique qui fait que dans certains contextes certaines variantes temporelles et / ou aspectuelles paraissent rejetables. Nous proposons de rendre compte de ce type d'inacceptabilité grâce au signe d'exclamation (!); c'est par exemple le cas des phrases (2a) et (2b) que nous empruntons respectivement à Vetters (1996: 115) et Kleiber (1993: 160):

(2) a. ILe 5 Juin 1989, Jules rentrait chez lui en 50 minutes.

réalité, l'indicatif est, des deux modes, le plus marqué puisqu'il comporte dans l'usage courant huit temps [...], le subjonctif seulement deux; la fréquence de leurs emplois n'est pas comparable, puisqu'ils apparaissent le plus souvent dans des contextes différents. ॥

Pour nous, cette hiérarchisation est non seulement non fondée, mais, en plus, elle a souvent servi d'alibi à l'étude de la syntaxe de l'IND. Nulle part, on ne dit que dans telle ou telle structure syntaxique l'IND est obligatoire. Or, ces structures existent. 
b. (le commandant ... se jeta sur l'interphone) et hurla qu'il avait à parler à $M$. Chisnutt.!Trois minutes plus tard, M. Brown ${ }^{3}$ se présentait chez le commandant.

Il ne s'agit pas ici à notre sens d'une simple notation mais d'une distinction capitale entre deux types d'inacceptabilité qui appartiennent à deux niveaux d'analyse distincts. En effet, à y voir de plus près, l'on se rend compte que les phrases (lb-d) sont inacceptables quel qu'en soit le contexte. La notion d'agrammaticalité dans leur cas reste tout à fait pertinente puisqu'il s'agit bien d'un rejet architectural. Ce n'est pas le cas, nous semble-t-il, des phrases (2) où l'on devrait parler plutôt d'inadéquation logico-sémantique qui, elle, contrairement à l'agrammaticalité syntaxique, opère aussi bien au niveau de la phrase qu'au niveau du (con)texte (cf. (2b) et Vetters 1993).

De façon tout à fait curieuse, un certain nombre de recherches syntaxiques relativement récentes, opérées surtout dans le cadre du modèle $G B$, en cherchant à relier l'interprétation temporelle des énoncés à leurs structures syntaxiques, vont à l'encontre de l'hypothèse défendue $\mathrm{ici}^{5}$ et qui nous semble, somme toute, assez triviale, du moins dans ses versions les plus relâchées. Héritant du nœud INFL de Chomsky (1981), qui mettait dans un même niveau toutes les données flexionnelles (mode, temps, aspect, personne, nombre et genre), ces recherches ne semblent pas avoir enregistré une quelconque hiérarchie entre la catégorie Mode, d'une part, et les catégories Temps et Aspect

3 Par opposition à Mr. Chisnutt qui, selon l'analyse de Molendijk (1990) reprise par Kleiber (1993), rend la phrase acceptable. Sans être totalement en désaccord avec l'analyse de Kleiber, il nous semble, quant à nous, que (2b) n'est pas totalement inacceptable, mais qu'elle nécessite un plus grand effort de récupération sémantique (dans l'esprit de Sperber \& Wilson (1989)) que la phrase correspondante avec Mr. Chisnutt.

$4 \quad$ Vetters (1996: 115, n. 38) précise qu'il a ajouté l'indication de l'année « pour éviter que la phrase $[(2 a)]$ ne reçoive une interprétation habituelle. " Autrement dit, l'inacceptabilité de l'exemple en question provient d'une inadéquation entre l'imparfait et les deux compléments temporels insérés de part et d'autre de la "principale» (avec l'un de ces deux compléments temporels, la phrase demeure acceptable)...

s Cf. par exemple Enç (1987), Guéron (1993) \& (1996), Guéron \& Hoekstra (1988). Ce n'est pas le lieu ici de revenir en détail sur ces travaux. Contentonsnous simplement de souligner que nous avons la nette impression que certaines de ces recherches n'ont pas toujours conscience qu'elles ne font que représenter syntaxiquement des données temporelles qui n'obéissent pas à la syntaxe. Or, on n'exige pas d'une représentation simplement qu'elle 'fonctionne'; il faudrait aussi qu'elle soit linguistiquement plausible. 
de l'autre. Résultat: on parle de la syntaxe des temps, mais pas (encore) de la syntaxe des modes!

\section{Modèle syntaxique: Gouvernement et Liage}

Pour rendre visibles les données pertinentes et les présenter d'une façon claire et économique, il est nécessaire de disposer d'un modèle syntaxique précis. Nous choisissons ici le modèle global présenté par Chomsky (1981, 1982 1986(a, b) \& 1991) et connu sous le nom de la Théorie du Gouvernement \& du Liage (GB). Il va sans dire que l'adoption d'un modèle ne signifie pas l'adoption de toutes les analyses qui y sont avancées, d'autant que ces dernières sont en l'occurrence loin d'être toujours homogènes et plusieurs versions concurrentes en sont souvent proposées pour rendre compte d'un même phénomène. Nous ne tenons pas non plus compte de toutes les modifications apportées par Pollock (1989), adoptées en partie et généralisées par Chomsky (1991), parce que d'une part, ces modifications ne jouent pas ici un rôle décisif et, d'autre part, parce qu'une version allégée ménagerait plus la clarté de l'exposé.

Une présentation sélective et sommaire de ce modèle est ici nécessaire. Nous commencerons par la présentation de la Théorie X-barre (Chomsky: 1970) qui considère toutes les catégories syntaxiques comme des catégories endocentriques, c'est-à-dire comme les projections maximales ( $\left.\mathrm{X}^{\prime \prime}\right)$ de leurs têtes $(\mathrm{X})$ lexicales ou fonctionnelles. Le système $\mathrm{X}$-barre peut prendre la forme des deux règles P-S (Phrase Structures Rules) suivantes:

(3) a. $\quad X^{\prime \prime} \longrightarrow \operatorname{Spec} X^{\prime} X^{\prime}$

b. $\mathrm{X}^{\prime} \longrightarrow \mathrm{X}(\ldots)$

où $\mathrm{X}$ (tête) est une variable valant pour toutes les catégories lexicales majeures $(\mathrm{N}, \mathrm{V}, \mathrm{P}, \mathrm{A})$ et, plus récemment, $\mathrm{C}(\mathrm{OMP})$ et $\mathrm{I}(\mathrm{NFL})$.

Cette extension aux catégories fonctionnelles du schéma X-barre s'inscrit dans le prolongement même de cette théorie qui, faisant abstraction à la fois de la nature catégorielle des termes lexicaux et de la nature lexicale (ou non) des catégories, se contente de mettre des formats structuraux à la disposition des 'termes' pour que ceux-ci, quels qu'ils soient, puissent développer leurs projections.

La Théorie X-barre étendue - si on ose l'appeler ainsi - ne répond pas simplement à une nécessité d'homogénéisation théorique interne, mais en plus, et surtout, émet un certain nombre d'hypothèses empiriquement falsifiables sur les phrases 'simples' (notées, pour le moment, $S$ ) et 'complexes' (notées, pour le moment, $S^{\prime}$ ) qu'elle considère comme les projections respectivement de $I N F L$ 
(et non plus de $V$ comme dans les états précédents du modèle chomskien) et de COMP, selon les deux règles $\mathrm{P}-\mathrm{S}$ provisoires suivantes:

(4) a. $\mathrm{S}^{\prime} \longrightarrow$ COMP S

b. $\mathrm{S} \longrightarrow \mathrm{N}^{\prime \prime}$ INFL V"

L'application du système $X$-barre aux phrases de type $S$ passe par la reconnaissance d'un niveau hiérarchique intermédiaire $(X$, en l'occurrence $I N F L$ ) entre la tête - INFL - et sa projection maximale (X") - S ou, donc désormais, $I^{\prime \prime}$ (que l'on peut noter aussi IP de Inflection Phrase). Les trois constituants à droite dans (4b) sont donc hiérarchisés, et le système de $\mathrm{S}$ peut être représenté grâce aux deux règles P-S suivantes:

(5) a. $\mathrm{S}=\mathrm{IP} \longrightarrow \mathrm{NP}$ INFL'

b. INFL' $\longrightarrow$ INFL V"

On obtient ainsi pour une phrase $S$ 'simple' la configuration (en crochets étiquetés) (6a) et la structure arborescente (6b) qui sont censées représenter le schéma syntaxique global d'une indépendante 'simple' en français:

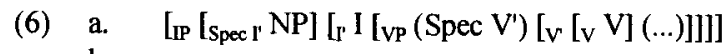

b.

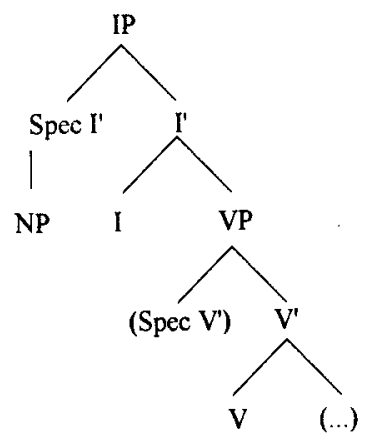

On admet généralement que le nœud I est composé d'une part d'un constituant Temps et d'autre part d'un constituant $A G R$. Ces deux constituants entretiennent un rapport étroit qu'on énonce habituellement comme dans la règle suivante:

(7) $\mathrm{I} \longrightarrow[ \pm$ Temps] $[ \pm \mathrm{AGR}] \quad(\Leftrightarrow \mathrm{Si}[+$ Temps], alors $[+$ AGR $])$

qui lie l'apparition du constituant Temps à celle d' $A G R$. Il s'agit là d'une donnée triviale pour une langue comme le français où le temps fini est nécessairement personnel. Mais, nous y reviendrons. 
D'autre part, on aura noté dans les représentations (6) l'existence de deux types de parenthèses: les premières, dans la branche droite de $V^{\prime}$, signifient simplement ici que l'existence d'un (ou de plusieurs) complément(s) éventuel(s) dépend du terme étiqueté $\mathrm{V}$ lui-même. La présence ou l'absence de compléments (ou plutôt de positions compléments) est supposée constante, en vertu du Principe de Projection $(P P)^{6}$ et en accord avec la sélection lexicale du verbe en question (le verbe donner sélectionne deux positions compléments, manger une position, dormir aucune... $)^{7}$. En revanche, les parenthèses en gras dans la branche gauche de VP signifient que cette position est facultative (voir sur ce point Chomsky (1986b)), comme toutes les positions de spécifieurs, à l'exception notable d'une seule: la position (Spec I'), c'est-à-dire la position syntaxique qui est censée accueillir le sujet de la phrase. Même si le $P P$ ne réussit pas à imposer l'existence constante d'une position sujet (notamment, parce que cette dernière peut accueillir des explétifs), on a été amené dans le modèle GB à poser le Principe de Projection Etendu (EPP) qui vise à rendre obligatoire la présence d'un sujet syntaxique. Là aussi, il a été proposé pour ce principe plusieurs formulations concurrentes dont les effets empiriques et théoriques ne sont pas identiques ${ }^{8}$. Admettons qu'on puisse l'énoncer ainsi:

(8) Clauses must have a subject.

$6 \quad$ Plusieurs versions concurrentes de ce principe sont proposées, qui n'ont pas les mêmes effets théoriques et empiriques. Ceci ne nous concernant pas directement ici, nous pouvons donner la version immédiatement lisible suivante qu'on trouve par exemple dans Chomsky (1981: tr.fr.: 61):

(i) Les représentations sont, à chaque niveau syntaxique (c'est-à-dire, en $F L$, en D-structure et en S-structure), des projections du lexique, en ce sens qu'elles respectent les propriétés de sous-catégorisation.

7 Il s'agit ici d'un résumé compact qui ne peut pas s'arrêter sur le problème, complexe, de l'unicité des positions appelées. Disons, pour simplifier, que, suite à Milner (1989) et contrairement à la tendance générale dans le modèle GB, nous distinguons entre la facultativité d'un terme et celle d'une position. Nous posons qu'une position maximalement appelée est toujours présente même si le terme qui doit l'occuper est absent. Cette analyse pose l'existence d'une catégorie vide, dans les positions appelées non-occupées phonologiquement, dès le niveau de D-structure. Cela nous permet de prévoir l'existence de phrases comme la vérité est, je n'ai pas mangé; j'ai déjà donné, etc., où les compléments sont absents. Voir Milner (1989) et Abouda (en préparation).

$8 \quad$ Voir, pour différentes options, Chomsky (1986a), Rouveret (1987) et Ouhalla (1994). Voir aussi la théorie du sujet grammatical de Milner (1989). 
Ce principe impose la présence dans toute phrase d'un sujet syntaxique, même en l'absence d'un sujet phonologique. En conséquence, la position (Spec I') peut être occupée par plusieurs types d'éléments:

(i) Un $N P$ lexical: c'est le cas des phrases à temps fini dans des langues comme le français ou l'anglais. Dans ce cas, c'est $A G R$, marqué [+], qui assigne le Cas Nominatif à ce $N P$, comme l'indique le principe suivant:

(9) NP $\longrightarrow[+$ nom] quand il est gouverné par et coïndicé avec AGR

(ii) Une trace (notée $t$ ): c'est le cas où le sujet présent dans sa position canonique en $\mathrm{D}$-structure est déplacé dans une autre position en S-structure comme dans l'exemple suivant:

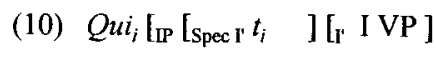

(iii) $P R O$, qui est considéré comme un NP vide (non sujet au Filtre sur le Cas) ayant une matrice de traits [+ Anaphorique, + Pronominal], contrairement aux autres catégories vides, ne doit pas être gouverné. C'est donc le candidat naturel à la position (Spec I') dans le cas général des phrases infinitives, caractérisées par l'absence du constituant AGR (cf. (7) supra. ) ou, selon d'autres analyses, par l'absence d'un élément plein dans le constituant AGR (cf. infra $\S 2.3$ ).

(iv) Un élément pro qui se distingue du PRO par le fait qu'il est gouverné par $A G R$. On admet en GB l'existence de cette catégorie vide pour rendre compte des langues à Sujet Nul (Pro-Drop languages), qui ont la particularité de pouvoir avoir un verbe fini, donc marqué $[+A G R]$, sans que la position syntaxique du sujet soit remplie par un élément lexical ${ }^{9}$, soit que le sujet est inversé, soit qu'il n'existe pas. C'est le cas général des grandes langues romanes à l'exception, dit-on, du français. Nous y reviendrons.

L'application du système X-barre aux phrases de type $S^{\prime}$ (règle (4a)) passe aussi par la reconnaissance d'un niveau intermédiaire entre le terme en positiontête, $\mathrm{C}\left(\mathrm{OMP}\right.$ ), et sa projection maximale $\mathrm{X}$ ", en l'occurrence $\mathrm{C}^{\prime \prime}$ (ou $C P$ ). Ce qui revient à reconnaître deux positions pré-IP distinctes, au lieu d'une seule comme cela avait été le cas dans le modèle de Bresnan-Chomsky datant des années 1970. La première position est celle du C(OMP)-tête qui accueille

Haegeman (1994: 455) note: « We say that a rich INFL can identify an empty category in the subject position while a poor INFL fails to do so. In other words the grammatical features of the subject can be recovered from those of INFL, specially from AGR, in languages with rich verb inflection. " 
principalement les subordonnants libres (au sens de Milner (1978)), à savoir si, que dit complétif et, suite à Huot (1981), de (qu'il ne faut pas confondre avec la préposition-homonyme). La deuxième position pré-IP est Spec $\mathrm{C}^{\prime}$ qui est la position qui accueille les constituants $q u{ }^{10}$ interrogatifs et relatifs: qui, que, quand, ò̀, etc. Vu les liens, mis en évidence par Milner (1973 \& 1978), entre les interrogatives-relatives et les exclamatives, nous pensons qu'il est possible de considérer que les constituants $q u$ - exclamatifs (et peut-être comparatifs) ${ }^{11}$ se déplacent aussi à la position $\mathrm{Spec} \mathrm{C}^{\prime}$.

Cette analyse, qui nous semble tout à fait plausible pour des raisons à la fois empiriques et théoriques ${ }^{12}$, revient à rejeter la règle (4a) en la remplaçant par le couple suivant de règles $\mathrm{P}-\mathrm{S}$ :

(11) a. $\mathrm{S}^{\prime}=\mathrm{CP} \longrightarrow \operatorname{Spec} \mathrm{C}^{\prime} \mathrm{C}^{\prime}$

b. $\quad \mathrm{C}^{\prime} \longrightarrow \mathrm{C}$ IP

On obtient ainsi les deux configurations suivantes ${ }^{13}$ comme schéma général d'une phrase de type S':

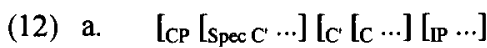

10 Il ne faut pas croire que cette appellation sténographie une quelconque propriété morphologique puisque le diagramme qu- n'est pas nécessairement présent à l'initiale de tous ces constituants (où, lequel, etc.).

11 Il nous semble que le modèle de Milner (1973 \& 1978), conçu dans le cadre de Bresnan-Chomsky, est directement transposable dans le cadre du modèle GB, modulo quelques petits arrangements. Malheureusement, notre intuition n'est appuyée pour le moment par aucune recherche récente sur la question. Quoi qu'il en soit, vu le principe (13) infra, l'analyse globale présentée ici est indifférente au traitement que l'on peut réserver aux exclamatives puisqu'elle permet de récupérer le trait pertinent, que le mot $q u$ - exclamatif se déplace (soit généré?) sous $\mathrm{C}$ ou sous $\mathrm{Spec} \mathrm{C}^{\prime} \ldots$

12 Les raisons empiriques essentielles sont fournies par l'existence de langues qui ne respectent pas le Filtre du COMP doublement rempli (Doubly Filled COMP Filter), i.e. où deux positions pré-IP sont pleines en même temps, comme dans la phrase suivante (vieil anglais): Men shal wel knowe who that $I$ am ='les hommes sauront qui je suis'. Les raisons théoriques sont très nombreuses, mais on peut évoquer principalement la Théorie du Mouvement (cf. Chomsky (1986b)).

13 Le IP dans ces deux représentations se développe bien entendu comme dans $(6 a, b)$. 
b.

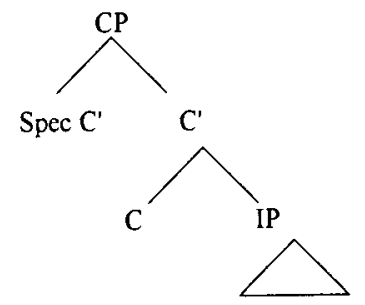

Avant de clore ce paragraphe, il nous reste à régler un dernier problème: comme $\mathrm{C}$ est considéré comme la tête de $\mathrm{CP}$, on devrait s'attendre à ce que les caractéristiques sémantiques de l'élément inséré sous $\mathrm{C}$ se projettent dans le $\mathrm{CP}$ en entier. Lorsque cet élément est un Complémenteur, cela ne pose aucun problème: pour prendre en exemples des complémenteurs anglais, il est facile de constater qu'à for correspond un $\mathrm{CP}$ infinitif, à whether et if correspond un $\mathrm{CP}$ interrogatif et à that correspond un $\mathrm{CP}$ déclaratif. Les caractéristiques du complémenteur sont donc directement projetées sur la phrase toute entière: on parle généralement dans ce cas de percolation du trait de tête. Or, concernant les éléments $q u$, nous avons dit ci-dessus qu'ils étaient insérés sous le (Spec $\mathrm{C}^{\prime}$ ) (et non pas sous $\mathrm{C}$ ). Dans ce cas, dans des phrases interrogatives de type que mange-t-il?, la position-tête de CP étant vide, on ne pourrait pas dire que le trait 'interrogatif' contenu dans la projection maximale CP soit transmis par la tête. Pour résoudre ce problème, nous évoquons le principe connu sous le nom de Spec-Head Agreement (Accord Spec-Tête). Ce principe, conçu à l'origine comme une relation d'accord en personne, genre et nombre, a été étendu suite à Chomsky (1986b) à toutes les autres relations entre une tête et son spécifieur. Il annonce d'une façon générale qu'un spécifieur et sa tête doivent partager (ou s'accorder en) les traits pertinents. On peut en donner ici la formulation suivante qu'on trouve par exemple dans Ouhalla (1994: 105):

(13) Spec-Head Agreement

A head $(\mathrm{X})$ and its specifier (Spec XP) must agree in relevant features.

Les traits pertinents en question, de même que leurs sous-traits éventuels, varient selon la nature catégorielle du terme-tête et aussi selon les langues ${ }^{14}$. La

14 Ainsi, par exemple, dans une langue comme le français, un déterminant partage avec le nom les traits de personne, de genre et de nombre. Dans d'autres langues, certains de ces traits (ou tous) sont absents. En français, le sujet partage avec INFL les traits de personne et de nombre. En arabe standard, dans cette même relation sujet-INFL (ou plutôt sujet-verbe, pour des raisons qui ne nous concernent pas ici, mais cela n'affecte pas l'argumentation en cours), il convient 
nature elle-même de ces traits n'est pas contrainte: elle peut être aussi bien phonologique, morphologique que sémantique. Si tel est le cas, on suppose que le même phénomène d'accord est valable dans les configurations (12) et c'est donc (13) qui permet à un constituant $q u$ de transmettre un trait (en l'occurrence, dans que mange-t-il?, un trait de type 'interrogatif', noté généralement [+WH] ou [+QU] en français) à la tête vide insérée sous $\mathrm{C}$.

Pour atteindre un degré de généralité adéquat, plusieurs traits ont été proposés (pour le français, cf. Milner (1978), Huot (1986 \& 1988), Contreras (1991), entre autres) pour distinguer entre les différents éléments $q u$ - et complémenteurs occupant les positions pré-IP. La distinction qui nous semble ici pertinente, i.e. qui aura des conséquences sur le choix du mode verbal, sera rendue par un trait lexical, $[ \pm T]$, qui oppose la conjonction que, élément sémantiquement vide et noté ici [- T], aux éléments, spécifiés $[+\mathrm{T}]$, qui ont comme caractéristique d'introduire soit (i) une idée de non-assertion: c'est le cas de la conjonction si (à la fois dans les hypothétiques et les interrogatives indirectes totales) qui suspend la valeur de vérité de la proposition qui suit ${ }^{15}$ et de tous les éléments qu- interrogatifs (que, qui, quand, lequel, ò̀, etc.); soit, au contraire, (ii) une idée d'assertion intensifiée: c'est le cas des éléments $q u$ exclamatifs: si, comme, combien, que, quel, etc.

Cette classification peut sembler a priori surprenante. Elle ne nous semble pas moins fondée: en réalité, on ne fait ici qu'opposer le domaine de l'assertion (qui constituerait une échelle allant de l'assertion intensifiée à l'assertion hypothétique, faible ou nulle, en passant par la question ${ }^{16}$ ) à un domaine où le

d'ajouter aux deux traits ci-dessus un trait de genre. Les valeurs elles-mêmes des différents traits peuvent varier. Ainsi, par exemple, le français oppose deux valeurs de nombre (singulier vs pluriel) là où l'arabe en distingue trois (singulier vs duel vs pluriel)...

15 Naturellement, l'opposition entre si et que complétif est un thème classique de la grammaire du français (cf., par exemple, Guillaume 1929 et Moignet 1974). Des recherches linguistiques relativement récentes ont cherché à rapprocher ces deux complémenteurs (cf. R.Martin 1983, H.Curat 1991 entre autres). Même si ces rapprochements nous semblent légitimes, l'analyse linguistique devrait, selon nous, garder les moyens de distinguer sémantiquement entre ces deux complémenteurs. Suivant en cela Milner (1989), nous pensons que le que complétif fonctionne comme un 'sas' qui ne joue de rôle que syntaxique et peut éventuellement dans certaines langues (comme l'anglais) ne pas apparaître. Ce n'est pas le cas, nous semble-t-il, de si qui, même s'il ne peut pas être syntaxiquement qualifié de subordonnant lié (au sens de Milner 1978), ne peut pas être qualifié de sémantiquement vide.

16 Poser une question, même globale, c'est, selon Anscombre \& Ducrot (1983) poser une "assertion préalable »; selon Martin (1984: 263; cité par Confais (1990)), " la question présuppose la vérité dans quelque monde possible ". 
trait [ \pm Assertif] n'est même pas pertinent, où l'on est en dehors de l'assertion (et de son contraire: la non-assertion). Si ce traitement est acceptable, le trait $[+\mathrm{T}]$ se réécrira $[ \pm$ Assertif], alors que le trait $[-\mathrm{T}]$ signalera un domaine, dont les contours restent à préciser, et où l'assertion, au sens général, n'est même pas pertinente. On pourra songer à réécrire [- T] comme un trait [Hors-Assertion]. ${ }^{17}$

\section{Phénomènes syntaxiques pertinents}

Avant de proposer les différentes structures syntaxiques des différents modes, il est primordial de s'arrêter sur trois problèmes majeurs de la syntaxe du français, à savoir le statut des clitiques sujets $(\S 2.1)$, l'inversion du sujet $(\S 2.2)$ et les différents composants de $I(\S 2.3)$.

\subsection{Statut des clitiques sujets}

Il a été démontré depuis Benveniste (1965) que les clitiques $(\mathrm{Cls})$ sujets en français se distinguent nettement des autres NPs lexicaux, à tel point qu'il semble aujourd'hui largement admis que les Cls n'ont pas le statut de Noms. Se pose alors la question primordiale suivante: les clitiques sujets occupent-ils la même position syntaxique que celle occupée par les NPs lexicaux sujets?

Un nombre important de données suggère que les Cls semblent, en cas d'inversion, plus proches du verbe que leurs correspondants lexicaux, comme le montrent les données suivantes, reprises de Kayne (1973):

(14) a. Que voulait-il manger?

a'. *Que voulait manger il?

b. *Que voulait ce garçon manger?

(15) a. Qu'a-t-elle fait?

b. *Qu'a cette femme fait?

$$
\begin{aligned}
& \text { b'. Que voulait manger ce garçon? } \\
& a^{\prime}, \quad \text { *Qu'a fait-elle? } \\
& \text { b'. Qu'a fait cette femme? }
\end{aligned}
$$

La même distinction semble être de rigueur en cas de mise en facteur du sujet, comme le font voir les données (16) et (17):

Nous sommes conscient que le rapport exact entre question, hypothèse et assertion est aujourd'hui au centre d'un débat qui est loin d'être terminé. Pour une discussion exhaustive des différentes positions sur la question de l'assertion, nous renvoyons à Confais (1990).

17 Il nous semble que cette opposition [ \pm Assertif] vs [Hors-Assertion], même si elle était approchée, quelquefois de très près, par certains grammairiens et linguistes (cf. Damourette \& Pichon, Huot (1988 \& 1991)), n'a jamais été exprimée très clairement. Elle se confond bien souvent avec l'opposition Assertif vs Non-Assertif, deux traits qui, même s'ils sont distinguables (et on a raison de les distinguer); appartiennent selon nous à un même domaine global... 
(16) a. * On a bu du bon vin et a mangé de la bonne viande.

b. On a bu du bon vin et on a mangé de la bonne viande.

c. On a bu du bon vin et mangé de la bonne viande.

(17) a. Pierre a lu du Wagner et a écouté du Berlioz.

b. Pierre a lu du Wagner et il a écouté du Berlioz.

c. Pierre a lu du Wagner et écouté du Berlioz.

Cela suggère que le clitique sujet, contrairement aux NPs lexicaux, ne peut être supprimé qu'avec la suppression de l'auxiliaire: les clitiques sujets sont attachés à l'auxiliaire, donc au verbe.

D'une façon plus générale, le français oral non seulement admet, mais semble même préférer, les énoncés où il y a à la fois réalisation du sujet lexical et du clitique sujet, et ceci dans l'ordre linéaire exclusif: $\mathrm{NP}-\mathrm{Cl}$ :

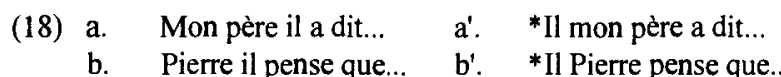

L'observation des courbes intonatives des exemples de type (18a-b) tend à démontrer qu'il n'y a pas de pause entre le NP lexical et le $\mathrm{Cl}$, ce qui rend difficile de considérer en syntaxe que l'on ait affaire à des cas de détachement.

Or, à y voir de plus près, des exemples comme (18), qui, à notre connaissance, n'ont pas réussi jusqu'à maintenant à trouver un traitement adéquat, posent des problèmes importants au modèle GB lui-même. Non seulement ils entrent en contradiction directe avec la Théorie du Liage (ils en enfreignent le Principe B), mais, en plus, ils ne semblent pas respecter le $\theta$ critère: pour chaque verbe on doit avoir normalement un seul $\theta$-rôle indirect (sujet) de disponible; or, ici, nous avons deux éléments pleins et référentiels qui sont disponibles pour un seul $\theta$-rôle assigné ${ }^{18}$.

Pour résoudre toutes ces difficultés et proposer un traitement unitaire et plausible des clitiques sujets, nous pensons qu'il est possible de soutenir que les clitiques sujets sont des morphèmes verbaux discontinus ${ }^{19}$. A ce titre, ils sont

18 Les mêmes exemples (18) interpellent le principe suivant de Milner (1989: 496):

(i) Dans un domaine, on ne rencontre pas deux fois les mêmes fonctions; on ne rencontre pas deux fois les mêmes rôles.

Mais là, on voit au moins dans quelle direction il faut chercher la solution.

19 Il ne faut pas croire que cette solution soit nouvelle: outre un certain nombre de grammairiens et linguistes (comme, semble-t-il, Jaeggli (1980) dont le travail reste malheureusement introuvable), H.Huot (1987) l'a avancée (mais l'a abandonnée depuis) en employant des termes très proches de ceux utilisés ici. Sauf que, travaillant dans le cadre du modèle GB, nous posons que le $\mathrm{Cl}$ est 
générés sous AGR (dans INFL), comme tous les morphèmes de personne. Outre que cette solution permet de rapprocher le français des autres grandes langues romanes (le français serait alors une langue "pseudo-pro-drop"), les avantages descriptifs et explicatifs qu'elle permet sont innombrables. Malheureusement, nous ne pouvons pas les développer ici. Signalons simplement que le contraste entre les exemples (16) et (17) trouve ici une explication satisfaisante. En effet, contrairement à la coordination entre VPs (cf. (16c) et (17c)) et entre IPs (exemples (16b) et (17b)) qui reçoit un traitement satisfaisant en GB, et demeure naturelle aussi bien avec un sujet lexical qu'avec un $\mathrm{Cl}$, le contraste entre (16a) et (17a) reste jusqu'à maintenant inexplicable. Godard (1989), discutant une proposition de Valin, a clairement démontré que dans des phrases coordonnées de type Jean a parlé avec Marie aujourd'hui et lui demandera un rendez-vous (exemple syntaxiquement analogue à (17a)), il n'y a pas coordination entre deux IPs mais une coordination entre deux INFLs' selon la configuration suivante:

(19) [IP [Spec I NP] [r [r a parlé avec Marie aujourd'hui] - et - [r lui demandera un rendezvous]]]

généré sous $A G R$, et non pas sous $V$ (comme chez Huot). Il ne s'agit pas d'une parade technique, mais d'un traitement qui, tout en demeurant plausible linguistiquement, permet de résoudre la plupart des difficultés rencontrées et de se prémunir contre les diverses critiques dont était susceptible l'analyse de Huot.

D'autre part, nous savons que le concept de morphème discontinu rencontre une certaine résistance. On peut présenter notre analyse différemment en disant que les Cls sont générés sous AGR, partie constitutive de INFL. Mais, comme AGR et INFL sont les nœuds où sont réalisés les morphèmes flexionnels, on comprendra que la nuance demeure simplement verbale. En fait, la linguistique, nous semble-t-il, malgré toutes ses déclarations, reste encore un peu prisonnière du mot. A ce propos, l'hypothèse du nœud INFL, opérée dans GB, est un progrès morphologique indéniable puisqu'on reconnaît que les morphèmes d'un mot puissent être réalisés dans des nœuds syntaxiques distincts. Nous ne faisons ici que prolonger cette logique: à la syntactisation de la morphologie, nous ajoutons la morphologisation de la syntaxe en reconnaissant que des 'mots' distincts puissent faire partie d'un même complexe morphologique (à charge de la morphologie de trouver une définition adéquate de la notion de complexe). Ajoutons que le paramètre 'Sujet Nul' est loin d'être clair à cause justement du rapport ambigu qui le lie au concept de mot, sous-jacent mais jamais avoué. Dans ces conditions, il n'est pas du tout étonnant que les mêmes dialectes italiens du Nord soient considérés à la fois comme des langues 'pro-drop' (Rizzi (1986)), et comme des langues 'non-pro-drop' (Safir (1986)). 
Telle quelle, cette analyse ne permet pas d'expliquer pourquoi des exemples comme (16a) sont exclus. Mais si l'on pose, comme nous le faisons ici, que le $\mathrm{Cl}$ est généré sous $\mathrm{INFL}$, on comprend les raisons de cette exclusion: le $\mathrm{Cl}$ appartenant à une position interne à INFL, donc a fortiori à INFL', ne peut pas être mis en facteur pour deux INFL's coordonnés...

Une plus grande démonstration dépasserait très largement le cadre de ce travail. Nous posons donc que le $\mathrm{Cl}$ sujet occupe en français une position interne à AGR et que la position (Spec $I^{\prime}$ ) peut être aussi bien vide qu'occupée par un NP sujet. Si elle est vide, vu le EPP (8), cette position sera occupée par un élément pro qui, comme on a pu le constater, est tout à fait naturel dans cette position.

En conclusion de ce paragraphe, nous dirons que la position (Spec I') peut être occupée par quatre types distincts de sujets: $N P$ lexical, $P R O$, pro et trace. Quant au $\mathrm{Cl}$, il sera considéré comme occupant une position interne à AGR (sous INFL). On verra que le paragraphe qui suit apporte un argument supplémentaire sur la nécessité de distinguer positionnellement $\mathrm{NP}$ et $\mathrm{Cl}$.

\subsection{L'inversion du sujet}

Ce paragraphe ne prétend absolument pas régler tous les problèmes, et ils sont très nombreux, de l'inversion sujet-verbe en français. Tout au plus s'agit-il d'un certain nombre d'indications, forcément sommaires ici, qui nous permettront d'entrevoir des solutions possibles à la question majeure qui nous préoccupe ici, i.e. la syntaxe des modes.

Revenons un instant sur la position C. Outre les subordonnants libres (ou complémenteurs) signalés ci-dessus, nous posons que cette position peut accueillir des verbes. Il s'agit d'une analyse aujourd'hui largement admise pour rendre compte de certaines inversions interrogatives en anglais et dans les langues germaniques en général, mais ne semble pas avoir été étendue au français. Quant à nous, sachant que la position C est une position à la fois noncanonique et polycatégorielle (cf. Milner (1989) à ce propos), nous ne voyons aucune raison empirique ou théorique ${ }^{20}$ qui empêche une telle possibilité de se réaliser dans certains cas, qu'il nous reste à déterminer.

Parmi ces cas, il faut d'emblée exclure des phrases comme:

(20) a. Mange-t-il la pomme?

b. L'aime-t-il sa petite femme!

20 En réalité, il y a une seule contrainte à respecter: la Théorie du mouvement (cf. Chomsky (1986b)) qui exige que l'élément sous $C$ soit une catégorie de rang zéro ( $\Leftrightarrow$ cette position ne peut pas accueillir des projections maximales). 
c. Est-il parti?

parce que s'il s'agissait de déplacer le verbe de I vers C, il serait impossible d'expliquer l'exclusion des phrases correspondantes avec un NP lexical (*mange Pierre la pomme?, * L'aime cet homme sa petite femme! ...). La seule solution plausible linguistiquement est de dire, comme cela est généralement admis, que c'est le $\mathrm{Cl}$ qui se déplace vers une position $\mathrm{P}$ à droite de $\mathrm{I}$.

Quelle est la nature de cette position P? Vu ce que nous avons dit au $\S 2.1$, la position canonique du $\mathrm{Cl}$ serait une position interne à $\mathrm{INFL}$, à gauche $\mathrm{e}^{21} \mathrm{du}$ constituant Temps. En cas d'inversion, la seule possibilité théorique (qui n'est pas différente en esprit de celle défendue par Kayne (1973) dans un cadre sensiblement différent) est de supposer que le $\mathrm{Cl}$ se déplace à droite dans une position adjointe à I, selon la configuration de C-Adjonction suivante ${ }^{22}$ :

(21) $\mathrm{XP} \longrightarrow(\mathrm{Y}) \quad \mathrm{XP} \quad(\mathrm{Y})$

En se déplaçant à droite, le $\mathrm{Cl}$ laisse une trace dans sa position d'origine, i.e. l'AGR à gauche du I. Quant à la position ( $\mathrm{Spec} \mathrm{I}^{\prime}$ ), elle peut être tout aussi bien pleine que vide. Dans ce dernier cas, nous l'avons dit, la position sujet est occupée par un élément pro. On obtient ainsi, pour une phrase comme (20a), la configuration suivante:

(22) $\left[_{\mathrm{IP}}\left[\left[_{\mathrm{Spec} \mathrm{I}}\right.\right.\right.$ pro $]\left[_{\mathrm{I}}\left[\left[_{\mathrm{I}}\left[\mathrm{I}\left[\mathrm{AGR} t_{i}\right]\left[_{\text {Temps }}\right.\right.\right.\right.\right.$ mange $\left.\left.\left.]\right]\left[\left[_{\mathrm{AGR}} i l_{i}\right]\right] \ldots\right]\right]$

Ce traitement, que nous ne pouvons malheureusement pas développer ici, permet d'expliquer l'existence de phrases comme:

(23) Pierre a-t-il mangé?

où, en analogie avec (22), l'on pose la structure suivante:

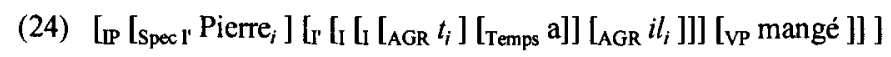

21 Cette analyse est compatible avec celle de Chomsky (1991): l'AGR dont on parle ici n'est rien d'autre que l'AGRS de Chomsky. Mais pour des raisons d'économie, nous ne développerons pas plus la structure interne de INFL connue sous son nom anglais: Split Infl Hypothesis.

22 L'adjonction n'est pas, comme on semble le croire parfois, en contradiction avec l'hypothèse de Préservation des Structures; voir sur ce point, Chomsky (1986b), Ouhalla (1994), Haegeman (1994), entre autres. 
Tous les cas d'inversion dans une interrogative (ou exclamative) totale trouvent, nous semble-t-il, une solution satisfaisante dans l'analyse avancée ici. En ce qui concerne l'inversion dans les interrogatives partielles, les données se compliquent et il ne nous est pas possible de les traiter dans le cadre de ce travail. Aussi prenons-nous simplement le cas des interrogatives avec que:

(25) a. Qu'a fait Pierre?

a'. Que fait Pierre?

b. Qu'a-t-il fait?

$b^{\prime}$. Que fait-il?

Concernant les phrases (25b-b'), l'hypothèse la plus plausible et la plus économique est de considérer que les Cls se sont déplacés à droite de I, dans une position adjointe, exactement comme dans les exemples (20) des interrogatives totales. Cette solution permet ainsi un traitement unitaire des diverses inversions du $\mathrm{Cl}$, que ce soit dans une interrogative (ou exclamative) totale ou dans une interrogative partielle.

Quant aux NPs (dans (25a-a'), il nous semble tout à fait possible de leur réserver un traitement analogue aux précédents. En effet, vu les liens évidents entre les phrases (25a-a') et leurs correspondantes avec $\mathrm{Cls}\left(25 \mathrm{~b}-\mathrm{b}^{\prime}\right)$, il serait inadéquat de poser dans leur cas que ce soit le verbe dans I qui monte dans la position C. Là aussi, on suppose donc que le NP se déplace à droite, quelque part, dans une position adjointe, $\mathrm{P}^{\prime}$. Nous savons déjà que cette position $\mathrm{P}^{\prime}$ ne peut pas être la même que celle des cas (25b-b') puisqu'on ne peut pas avoir une interrogative analogue linéairement à (25b) avec un NP: *Qu'a Pierre fait? Le participe passé, qui demeure dans une position interne à VP, suggère que la position $\mathrm{P}^{\prime}$ ne peut être qu'une position adjointe à VP. Plusieurs données dans les langues les plus diverses (comme l'italien) plaident en faveur de cette possibilité.

Nous proposons donc pour une phrase (25a') la S-structure suivante:

(26)

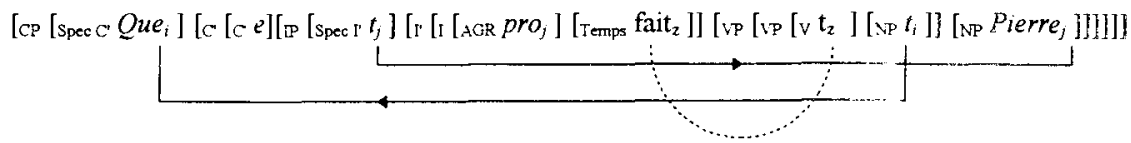

où les différentes relations de mouvement sont indiquées par la co-indiciation entre la trace et l'élément plein dans sa position finale en S-structure ${ }^{23}$. Nous reviendrons sur d'autres cas d'inversion lors de l'inventaire opéré ci-dessous infra $(\S 3)$.

23 Il y a dans la configuration (26) une relation de co-indiciation qui dénote un mouvement dont on n'a pas parlé parce qu'il est bien connu: la montée de $\mathrm{V}$ à I (V-raising): indice $z$. 


\subsection{Le noud I}

Tout en notant le caractère intuitif de $(5 b)$, nous aimerions souligner l'insuffisance du traitement que réserve $G B$ aux différents morphèmes flexionnels et ceci même dans le cadre des récents développements de la théorie ${ }^{24}$.

En accord avec l'hypothèse de départ que nous avons énoncée dès les premières lignes de ce travail - l'invisibilité des temps et des aspects pour la syntaxe -, nous croyons que $\mathrm{GB}$, ainsi que toute syntaxe positionnelle, est condamnée soit à noter d'une façon ad hoc des données aspectuelles et temporelles sans les expliquer, soit à énoncer des principes généraux trop coûteux et très peu explicatifs. Si une règle comme (7) fonctionne convenablement et ne contredit pas notre intuition, c'est justement parce qu'elle ne parle pas de temps, mais de modes. Y parler du trait $[ \pm$ Temps], c'est employer une terminologie abusive, voire erronée, très loin de pouvoir rendre compte de toute la complexité de la morphologie française. En effet, (7) suggère que le contenu de AGR soit déterminé en fonction du temps. Or, il existe en français des modes (certainement l'IMPé(ratif) et peut-être le $S U B$ (jonctif), au moins en français moderne) qui ne varient pas temporellement mais qui s'accordent avec le sujet en personne et en nombre. En effet, pour nous, mais en cela ne nous faisons que suivre la plupart des analyses modernes, les variations entre les formes simples et composées de l'IMPé, ainsi que les variations entre le SUB "présent" et le SUB "passé" ne dénotent pas des différences temporelles mais des différences aspectuelles (indépendamment de la valeur que l'on peut donner à cette variation aspectuelle). Or l'aspect grammatical lui-même ne peut pas constituer un critère apte à capter des différences en rapport avec AGR puisque tous les modes étudiés dans ce travail - y compris l'INF - connaissent en français la variation - aspectuelle - entre une forme simple et une forme composée.

A propos des temps, il semble que l'on est aujourd'hui presque unanime pour considérer que seul l'IND connaît véritablement des variations strictement temporelles ${ }^{25}$. Ces positions theoriques - qui sont aujourd'hui largement

24 Ainsi, par exemple, la Split INFL Hypothesis qui décompose INFL en $T P$ (Tense Phrase) et $A G R P$ poursuit en quelque sorte la même idée de départ de Chomsky (1981), sans opérer la moindre hiérarchisation entre les morphèmes temporels, aspectuels et modaux.

25 Plusieurs sont aujourd'hui les linguistes qui pensent que, d'une part, l'IMP et le $P Q P$ du $S U B$ sont des formes propres à l'écrit et à un style plutôt soutenu et peuvent être considérées comme des formes mourantes et, d'autre part, que si ces formes sont employées, elles ne renvoient pas à une temporalité 
partagées - sont résumées dans le tableau (27) dans lequel le signe "+" se lit « varie en » et le signe "-" « ne varie pas en » (aspect / temps / personne):

(27)

\begin{tabular}{|c|c|c|c|}
\hline Mode & Aspect & Personne & Temps \\
\hline INF & + & - & - \\
\hline IMPé & + & + & - \\
\hline SUB & + & + & - \\
\hline IND & + & 2 & + \\
\hline
\end{tabular}

Nous pouvons donc dire que les catégories Temps et $A G R$ ne sont pas obligatoirement présentes: cela dépend d'une façon cruciale du type de mode auquel nous avons affaire. En s'inspirant d'un modèle comme le HPSG, nous pourrons proposer, pour chacun des modes isolés, une structure de traits prédéfinis, comme en (28):

(28) Mode $=I M P e ́$

$$
\begin{array}{ll}
\text { Mode }=I M P e ́ & \text { Mode }=I N D \\
{[\text { Aspect }=\mathrm{x}]} & {[\text { Aspect }=\mathrm{x}]} \\
{[\text { Personne }=\mathrm{y}]} & {[\text { Personne }=\mathrm{y}]} \\
\text { [Nombre }=\mathrm{z}] & {[\text { Nombre }=\mathrm{z}]} \\
& {[\text { Temps }=\mathrm{t}]}
\end{array}
$$

$$
\begin{array}{ll}
\text { Mode }=S U B & \text { Mode }=I N F \\
{[\text { Aspect }=\mathrm{x}]} & {[\text { Aspect }=\mathrm{x}]} \\
{[\text { Personne }=\mathrm{y}]} & \\
{[\text { Nombre }=\mathrm{z}]} &
\end{array}
$$

Cela suggère de réécrire le nœud I comme en (29):

(29) I

$$
\longrightarrow \text { Mode Aspect (AGR) (Temps) }
$$

La règle (29) néc essite quelques précisions, au moins rapides:

(i) l'ordre des différents constituants est une question empirique qu'il reste à préciser $^{26}$;

(ii) d'une façon générale, et pour des raisons en rapport avec l'acquisition du langage, le modèle GB préfère des branchements binaires; or, ici, la règle (29) contient quatre constituants. Il serait possible de proposer une structure binaire: mais cela suppose une hiérarchisation préalable des différents constituants de (29). La question reste pour l'instant ouverte puisqu'elle dépasse de beaucoup l'objectif de cette étude. Signalons simplement qu'un module morphologique

(chronologique ou textuelle) mais paraissent plutôt comme un archaïsme (cf. la règle de la concordance des temps)...

26 Voir, par exemple, Pollock (1989) et Chomsky (1991) pour un débat sur l'ordre $A G R$ - Tense. Nous pensons quant à nous que cet ordre ne peut faire l'objet d'aucun principe de GU et devrait être considéré comme variable pour une langue (ou une famille de langues) donnée. 
autonome nous semble nécessaire pour rendre compte de ces données et qu'il n'est pas du tout évident que ce module puisse avoir des représentations analogues formellement aux autres représentations en D-structure, S-structure et $\mathrm{F}$ (orme) $\mathrm{L}$ (ogique).

A la fois parce que la syntaxe n'a pas grand chose à dire sur les temps et sur l'aspect grammatical ${ }^{27}$, et pour les raisons (i) et (ii), nous nous proposons, dans le cadre de cette étude, de ne pas entrer dans les détails de la constitution interne de I: on se contentera donc de le réécrire sous forme d'une structure de traits à l'allure suivante:

(30)

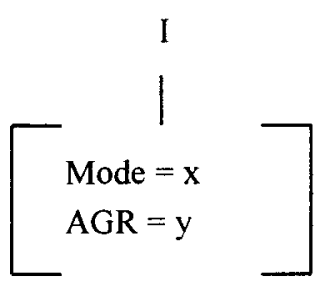

(iii) la catégorie mode se réécrit en prenant sa valeur dans la liste des quatre modes étudiés ici ${ }^{28}$ :

Mode $=\{I N F / I M P e ́$ /SUB $/ I N D\} ; " / "$ étant le signe de la disjonction.

Enfin, mises entre parenthèses, les composantes $A G R$ et Temps sont, comme on l'a montré ci-dessus, optionnelles. Pour l'obtention d'une généralisation descriptive adéquate, on pourrait songer à poser l'existence axiomatique de la position AGR dans tous les cas (comme dans (30)), quitte à ce que cette position soit vide dans un certain nombre de structures.

\section{Localisation syntaxique des modes verbaux}

Après ce survol des données essentielles qui vont jouer un rôle crucial dans l'apparition des différents modes, nous pensons avoir présenté le minimum d'outils indispensable pour opérer l'examen d'un cas particulier, celui de la phrase indépendante en français ${ }^{29}$.

27 Ce n'est pas le cas, nous semble-t-il, de certains phénomènes rangés sous la catégorie de l'aspect lexical et qu'on peut probablement qualifier de syntaxiques: cf. boire du vin vs boire un verre de vin; manger vs manger une pomme, etc.

28 On laissera ici ouverte la question du statut des participes.

29 Mais nous avons des raisons de croire que les variables syntaxiques isolées dans cet exposé jouent également un rôle important dans l'apparition des différents modes dans les structures complexes. 


\section{A. La Phrase ne contient pas un mot qu- ni un complémenteur}

\section{A.I. Sans Sujet phonologique}
(31) a
b. *Parte!
c. Soit!
(33) a. *Vient
b. *Partirait

Donc, dans ce cas précis, seuls les modes INF et IMPé sont possibles, à l'exclusion de tous les autres modes ${ }^{30}$. Peut-on amener les différences entre ces énoncés à l'IMPé et ceux à l'INF à une différence syntaxique? Il semble que oui. On se rappelle en effet que, dans les infinitives, étant donné le EPP (8), la position (Spec I') est occupée par un élément vide, PRO. Parallèlement, AGR est négatif, ce qui est tout à fait compréhensible sémantiquement puisque l'INF, en tout cas dans ce genre de structures, paraît renvoyer à un procès virtuel, indéterminé. Nous proposons donc de rendre compte des structures infinitives de ce type grâce au schéma:

\footnotetext{
30 Le $S U B$ est exclu avec tous les verbes, sauf avec le verbe être $(31 \mathrm{c})$. Mais il convient de préciser que non seulement être est la seule unité lexicale verbale possible dans une telle structure, ce qui est en soi une contrainte extrêmement forte, mais, en plus, il n'y est possible qu'avec la troisième personne du singulier ("Sois / "Soient), au temps "présent" (l'IMP et le PQP de SUB sont exclus: *Fût! / *Eût été! ) et à l'aspect inaccompli ( *Ait été! ). Syntaxiquement parlant, l'absence d'un sujet peut être ici considérée comme un indice suggérant qu'il ne s'agit pas d'une forme verbale, mais plutôt d'une forme figée.
} 
(35) Structure syntaxique de l'INF-1

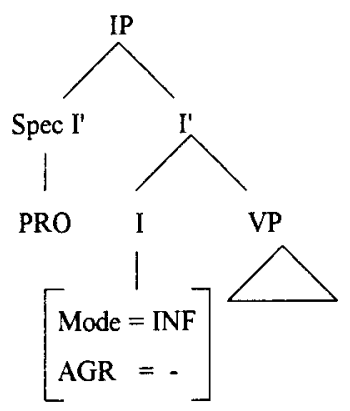

Contrairement à l'INF, le mode IMPé est un mode personnel. L'IMPé ne varie pas seulement en aspect, comme c'est le cas de l'INF, mais aussi en nombre et en personne (et même en genre dans une langue comme l'arabe standard). Il a ainsi un AGR riche. Il nous faudra concilier entre le fait qu'aucun élément lexicalement réalisé (que ce soit sous forme d'un NP ou d'un $\mathrm{Cl}$ ) ne peut jouer le rôle de sujet dans une impérative, et le principe (8) qui exige de toute phrase d'avoir un sujet. Il est donc nécessaire d'imaginer une catégorie vide qui va jouer le rôle syntaxique de sujet dans une phrase à l'IMPé. Le candidat le plus naturel à cette fonction n'est autre que pro. On se rappelle en effet que la condition sine qua non à l'existence de pro est l'existence d'un AGR riche. Or l'AGR d'une IMPé, comme on vient de le voir, est riche. Si ce traitement est acceptable, on aura pour l'IMPé la structure syntaxique suivante:

(36) Structure syntaxique de l'IMPé

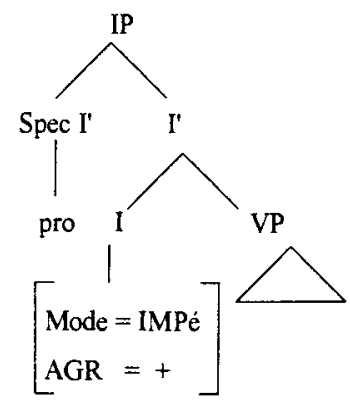

Ce traitement syntaxique nous paraît plausible et prépare le terrain à une interprétation logico-sémantique du mode IMPé. En effet, est-ce un hasard si les impératives ne supportent pas d'être enchâssées à un élément supérieur? Cette dernière caractéristique exclusive de l'IMPé peut être rapprochée d'une autre caractéristique, non moins intéressante, à savoir que l'IMPé ne supporte 
pas les troisièmes personnes, personnes qui appartiennent, par définition, à une autre sphère que celle du hic et nunc. Il semble ainsi que l'absence de sujet dans les impératives est due au fait que ces phrases se réfèrent à une situation discursive concrète, où le locuteur s'adresse à un (ou à des) interlocuteur(s) présent(s) dans une sphère commune: les morphèmes flexionnels qui s'attachent au verbe suffisent largement à déterminer la référence de pro, contrairement au cas des INF, dont l'absence du sujet peut être expliquée par la virtualité de la situation, une virtualité confirmée par l'absence des morphèmes flexionnels...

\section{A.II. Avec Sujet}

A.II. 1. Sans Inversion

L'IMPé et l'INF sont impossibles dans les constructions de ce type. Observons alors les exemples (37) à l'IND et (38) au SUB:

(37) a. Je voudrais quatre souris blanches.

b. Marie venait tous les jours...

(38) a. Le Ciel fasse que vous ayez raison! b. Dieu te bénisse!

Les exemples (38), et nous ne serons pas les premiers à l'affirmer, constituent des exemples non seulement archaïques et minoritaires, mais, en plus, extrêmement contraints: ils appartiennent presque exclusivement, en français moderne, à une classe sémantique particulière et leurs NPs lexicaux sont généralement de types $\mathrm{Ciel}$, Dieu, etc. Il ne serait donc pas impossible de leur réserver un traitement particulier: nous songeons à un trait lexical particulier qui serait attaché au NP sujet. Il n'est d'ailleurs pas étonnant à ce propos qu'aucune phrase de type (38) ne soit acceptable avec un $\mathrm{Cl}$. Dans le cas général (absence d'un trait spécifique contraignant le sujet et qui proviendrait, selon nous, du trait [-T] associé à la position $\mathrm{C}$ vide), nous posons que l'IND est le seul mode possible dans cette structure. Il sera dit obligatoire. La structure syntaxique d'une indicative de ce genre aura, mutatis mutandis, la même composition structurale que celle de l'IMPé, à la différence notable suivante: la position (Spec I') est ici occupée par un NP.

\section{A.II.2. Avec Inversion: \\ a. Le Sujet est un NP lexical}

Les données pertinentes sont les suivantes: 

(39) a. Mange la pomme, Pierre!
b. *Mange Pierre la pomme
(41) *Venir Pierre.
(40) a. *Vient Pierre?
b. *Vivrait le Roi!
(42) a. Vive le Roi!
b. Vienne l'été!
c. Soit un triangle $\mathrm{ABC}$.

Ces données suggèrent que dans la structure syntaxique en question, seul le SUB soit possible. Il n'en est rien, puisqu'un certain nombre d'énoncés de ce type peuvent être à l'IND. Examinons les exemples suivants:

(43) a. Dans la forêt vivait un vieil ermite

b. Dans la boitte étroite [...] ronflait, comme une grosse mouche, un ventilateur $^{31}$

c. Dans mon jardin poussent des plantes exotiques

d. Par la fenêtre ouverte, entrait une odeur de nuit et de fleurs

En comparant les exemples du SUB à ceux de l'IND, l'on se rend facilement compte qu'ils ont des caractéristiques syntaxiques diamétralement opposées ${ }^{32}$. Deux différences majeures méritent d'être soulignées ici:

(i) contrairement à leurs correspondantes au SUB, les phrases (43) admettent l'explétif $i l$ dans la position (Spec I') ${ }^{33}$ : cf. Dans la forêt il vivait un vieil ermite; Dans mon jardin il pousse des plantes exotiques...

(ii) contrairement aux exemples (43), qui sont naturels sans l'inversion, les exemples au $S U B$ (comme en (42)) ne sont plus acceptables si on annule l'inversion du sujet (cf. * Le Roi vive!) $)^{34}$.

31 Les données (b-d) sont empruntées à Tasmowski \& Willems (1987).

32 Entre autres différences, on peut signaler celles-ci: (i) contrairement aux exemples (43), qui semblent exiger systématiquement un circonstanciel (surtout de nature locative) antéposé, les exemples au $S U B$ sont agrammaticaux s'ils sont accompagnés d'un circonstanciel ouvrant la phrase; (ii) contrairement aux exemples au $S U B$, qui exigent que le $N P$ lexical sujet soit déterminé, les exemples à l'IND semblent exiger du sujet qu'il soit indéfini (ou générique) (cf. *Vive un Roi! )...

33 Nous pensons que l'explétif $i l$ n'est pas un élément de AGR: il ne s'agit pas d'un morphème de personne. Aucun argument positif ne semble pouvoir appuyer cette hypothèse. On se conente donc de signaler ici qu'aucun de nos arguments avancés à l'appui de l'hypothèse de la génération des Cls sous AGR n'est valable à propos de l'explétif $i l$.

34 Nous avons noté une seule exception: face à Fasse le Ciel que vous ayez raison! on peut avoir Le Ciel fasse que vous ayez raison. Cet exemple ne pose à notre avis aucun problème particulier: il peut tout à fait naturellement être traité comme un cas (avec inversion) de ceux qui sont étudiés sous A.II.1 (cf. (38)), c'est-à-dire en termes de contraintes affectant le NP. 
La donnée (i) suggère que là aussi, dans les exemples (43), on assiste à un déplacement du NP sujet dans une position adjointe à droite du VP, exactement comme dans les cas de l'interrogation étudiée dans le $\S 2.2$ (cf. (25)).

Mais est-ce le cas des exemples (42) au SUB?

Nous ne le pensons pas. Au moins deux indices suggèrent en effet que dans les phrases (42), c'est plutôt le verbe qui quitte sa position d'origine, i.e. sous I, pour aller vers la position $\mathrm{C}$ :

(i) L'impossibilité d'avoir dans les phrases (43) un explétif suggère que les NPs (Roi, triangle, etc.) puissent occuper la position (Spec I');

(ii) L'impossibilité du tour correspondant sans inversion (*Le Roi vive; *L'été vienne, etc.) ne permet pas de parler d'inversion du sujet. Il serait plus plausible de poser que le verbe, montant de $\mathrm{V}$ à I pour prendre ses affixes, n'y trouvant pas suffisamment d'attaches, continue sa montée jusqu'à la position $\mathrm{C}$, donnant ainsi lieu à un step-by-step movement respectant tous les principes gouvernant ce type de mouvement.

Si ce traitement est acceptable, on pourra proposer pour les phrases (42) la S-structure (44) et pour les phrases (43) la S-structure (45):

(44) Une Structure Syntaxique du $S U B-1^{35}$

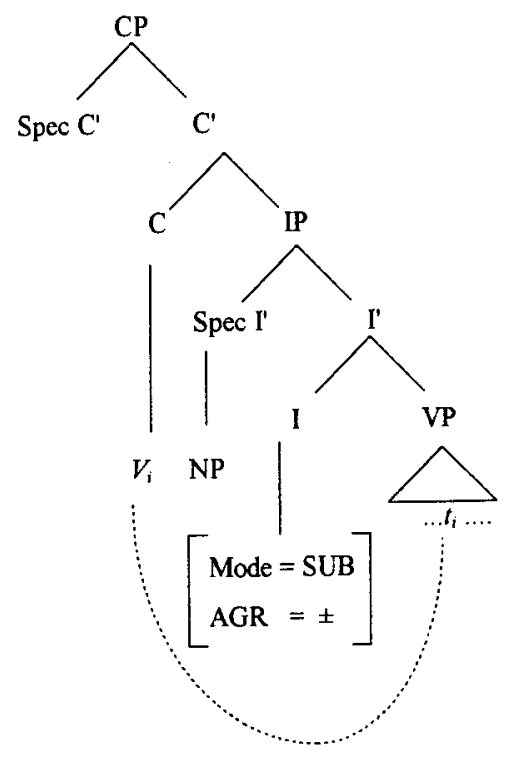

35 La notation "AGR $= \pm$ " vise à rendre compte du fait que l'accord entre le verbe et le sujet n'est pas systématique puisqu'on peut aussi bien écrire «Vive les Alliés! » que « Vivent les Alliés! ». 
(45) Structure Syntaxique de I'IND-2

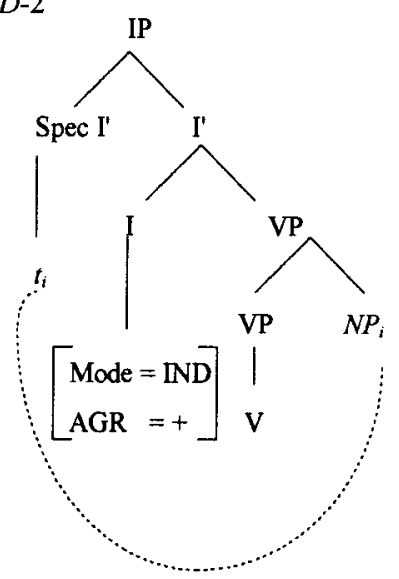

Il serait possible de relier ces différences syntaxiques entre les structures au SUB et les structures à l'IND à une différence de type logico-sémantique. En effet, des exemples comme (43) à l'IND appartiennent, dans leur majorité, au plan de récit (au sens de Benveniste): deux indices semblent suggérer cette direction: d'une part, ces tours préfèrent le temps du passé, notamment l'IMP (le PR est possible, mais il semble avoir systématiquement une valeur de PR du récit), et, d'autre part, ils me semblent comparables, certainement sur le plan sémantique, et peut-être aussi sur le plan syntaxique, au célèbre Il était une fois, l'IMP d'ouverture. A l'opposé, les exemples (42) au SUB ne s'emploient que dans le discours (le fait qu'ils ne supportent ni l'indéfini ni le circonstanciel est un indice significatif qui va dans ce sens)...

\section{b. Le Sujet est un Clitique}

Avec inversion du $\mathrm{Cl}$ sujet, seul l'IND semble possible:
(46) a. Vient-il?
b. Serait-il en colère?
(47) a. Est-il aimable!
b. L'aime-t-il sa petite femme!

Les phrases de ce type sont en effet très majoritairement exclues au subjonctif:
(48) a.
*Vive-t-il?/!
b. *Construise-t-il une maison!/?

On peut toutefois trouver des exemples comme les suivants:
(49) a. Ainsi soit-il!
b. Puisse-t-il être reçu! 
Mais il faut noter que dans (49a), la forme verbale est figée temporellement, aspectuellement, en personne et en nombre, sans parler du fait que seul un clitique (inversé) y est admis en guise de sujet. Aussi son statut verbal pourraitil être raisonnablement mis en cause ${ }^{36}$. Quant à la phrase (49b), suite à l'explication diachronique avancée par Wagner (1939), on pourrait songer à considérer les modaux pouvoir et devoir comme des cas particuliers qui peuvent être soit au subjonctif (il s'agit alors d'une survivance de l'état ancien de la langue), soit à l'indicatif. Dans le cas le plus général (non marqué), l'IND sera donc dit obligatoire et on aura pour ces structures le schéma syntaxique proposé ci-dessus dans (22).

\section{B. La Phrase contient un mot qu- (interrogatif / exclamatif) ou une conjonction}

Quelles que soient les structures considérées, l'IMPé est ici exclu. Pour les autres modes, il faut considérer les deux sous-cas suivants:

\section{B.I. Le mot qu- (ou le complémenteur) a le trait [士 Assertif]}

Soit les données suivantes:
(50) a. *Que fasse Pierre?
b. * Où partes-tu?
(52) a. Que faire?
b. Où aller?
c. Pourquoi partir?
(51) a. *Que soit-il beau!
b. *Que soit bête ce garçon!
(53) a. Que fait Pierre?
b. Oủ partirait-il?
c. Que n'écrit-il en prose!
d. Que ne l'ai-je su!
e. Qu'il était mignon!

On peut donc dire que seuls les modes INF et IND sont possibles dans les structures (B.I). Or, nous avons déjà posé la différence entre ces deux modes en termes syntaxiques: la structure de l'INF considérée ici ne se distingue de (35) que par l'existence d'une position (Spec $C^{\prime}$ ) remplie par un élément interrogatif. Quant aux phrases (53), elles ont une structure syntaxique très comparable à celles étudiées dans le $\S 2.2$.

36 Signalons tout de même l'existence de tours, apparaissant dans un style qualifié à juste titre par Le Goffic (1993: 502) de " recherché et archaïsant », de types: fussé-je; fût-il; eût-il, etc. 
B.II. Le complémenteur a le trait [- T] (ou [Hors-Assertion])

Dans ce cas précis, seul le SUB semble possible:

(54) a. *Qu'il part

b. *Que partir
(55) a. Qu'il parte!

b. Que ces hommes partent sur-le-champ!

Il sera dit obligatoire.

Nous aurons pour ces structures le schéma syntaxique suivant:

(56)

Structure syntaxique du SUB-2

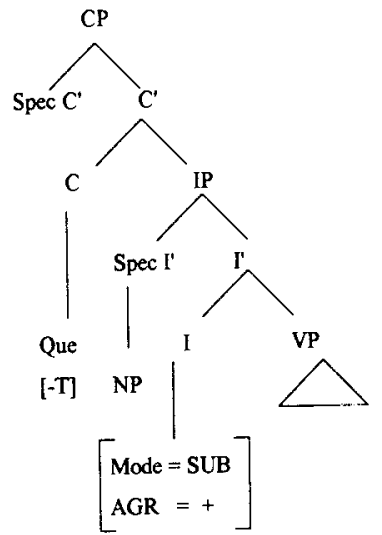

\section{En guise de conclusion}

Ce travail a tenté de ramener systématiquement les différences entre des énoncés ayant des modes distincts à des différences syntaxiques. Même si l'on ne prétend pas résoudre tous les problèmes, notamment techniques, relatifs à la représentation syntaxique des différentes structures considérées, la possibilité même d'une telle tentative nous semble significative. En effet, les modes, quels qu'ils soient, et seulement les modes, à l'exclusion des temps et des aspects (grammaticaux), ont des comportements visibles en syntaxe (fortement lexicalisée). Si nos hypothèses sont recevables, le programme de recherche que l'on inaugure ici pourrait avoir des conséquences empiriques intéressantes: la localisation syntaxique des modes verbaux pourrait nous aider non seulement à déterminer le sens de chacun de ces modes, approche parfois tentée dans ce travail d'une façon un peu elliptique, mais aussi à nous prononcer sur certaines formes verbales dont le statut modal et / ou temporel reste encore discuté. On songe notamment au conditionnel. L'observation des structures syntaxiques du conditionnel montre clairement qu'à chaque fois que l'on a affaire à cette forme verbale, on peut avoir, exactement dans la même structure syntaxique considérée, au moins un autre temps de l'IND. Il n'est nulle part obligatoire, 
nulle part imposé par des données strictement syntaxiques. Le conditionnel n'est pas visible pour la syntaxe: il s'agirait d'un temps... de l'indicatif.

Un certain nombre de faits viennent nous confirmer:

- Le mode est très généralement imposé par la structure syntaxique vs temps (si un mode est possible dans une structure syntaxique, toutes les variantes temporelles et aspectuelles de ce mode le sont également) qui reste selon nous une donnée discursive.

- Le mode est une donnée intra-phrastique (aucun mode n'est imposé par une phrase voisine ou même par une proposition en lien lâche) vs temps (cf. les notions d'anaphore temporelle, de cohérence et, par exemple, le conditionnel du style indirect libre).

- En cas de subordination, le mode est hérité de la proposition supérieure vs temps (critique de l'hypothèse de la concordance des temps: dans une subordonnée, les temps sont interchangeables)...

\section{Références}

Abouda, L. (1995). Le Conditionnel: temps ou mode? Arguments syntaxiques, Journée de Linguistique du CBL, Courtrai, 20 mai 1995. [à paraître dans Revue romane].

Abouda, L. (en préparation). Syntaxe et sémantique du conditionnel en français moderne, Thèse, Paris 7.

Benveniste, E. (1965). L'antonyme et le pronom en français moderne, in: Problèmes de linguistique générale, Paris: Gallimard, II, 1974, 197-214.

Bonnard, H. (1974). Les Axiomes temps et mode, Le Français moderne 42: $72-$ 89.

Chomsky, N. (1981). tr.fr. (1991). La Théorie du Gouvernement et du Liage, Paris: Seuil.

Chomsky, N. (1982). tr.fr. (1987). La nouvelle syntaxe, Paris: Seuil.

Chomsky, N. (1986a). Knowledge of language: its Nature, Origin and Use, New York: Praeger.

Chomsky, N. (1986b). Barriers, Cambridge, Mass.: MIT Press.

Chomsky, N. (1991). Some Notes on Economy of Derivation and Representation, in: R. Freidin (éd), Principles and Parameters in Comparative Grammar, Cambridge, Mass.: MIT Press.

Confais, J.-P. (1990). Temps, Mode, Aspect, Toulouse: Presses Universitaires de Mirail.

Contreras, H. (1991). On the position of Subjects, in: Susan D. Rothstein, (éd), Syntax and Semantics 25, Perspectives on Phrase Structure: Heads and Licensing, San Diego, California: Academic Press. 
Curat, H. (1991). Morphologie verbale et référence temporelle en français moderne, Genève: Droz.

Enç M. (1987). Anchoring conditions for tense, Linguitic Inquiry, 18, 633-657.

Godard, D. (1989). Empty Categories as Subjects of Tensed Ss in English or French?, Linguistic Inquiry 20: 497-506.

Guéron, J. (1993). Sur la syntaxe du temps, Langue française 100: 102-122.

Guéron, J. (1996). Cohérence et économie dans la grammaire du temps: remarques sur la variation des structures temporelles, Cahiers Chronos 1: $59-78$.

Guéron, J. \& Hoekstra, T. (1988). Les Chaînes-T et les verbes auxiliaires, Lexique 7: 61-85.

Guillaume, G. (1929). (éd. 1965). Temps et verbe, Paris: Honoré Champion.

Haegeman, L. (1994). Introduction to Government and Binding Theory, Oxford: Blackwell.

Huot H. (1981). Constructions infinitives du français: Le subordonnant "de", Genève: Droz.

Huot, H. (1986). Le Subjonctif dans les complétives: subjectivité et modalisation, in: M. Ronat \& D. Couquaux, (éds), La Grammaire modulaire, Paris: Minuit, 81-111.

Huot, H. (1987). Morphosyntaxe du verbe français et inversion du clitique sujet, Travaux de linguistique 14/15: 159-176.

Huot, H. (1988). Quelques conditions d'apparition du subjonctif: la notion de classifiance et le trait [ $\pm \mathrm{QU}]$, in: Recherches nouvelles sur le langage, DRL, Paris 7.

Huot, H. (1991). Quelques aspects syntaxiques de la non-assertion, Actes $d u$ XVIIIe Congrès International de Linguistique et de Philologie Romanes, t. II, Université de Trèves (Trier): Tübingen, Max Niemeyer Verlag, 389401.

Kayne, R. (1973). L'Inversion du sujet en français dans les propositions interrogatives, Le Français moderne, 41: 10-42; 125-151.

Le Goffic, P. (1993). Grammaire de la phrase française, Paris: Hachette.

Martin, R. (1983). Pour une logique du sens, Paris: PUF.

Milner J.-C. (1973). Arguments linguistiques, Paris: Maison Mame.

Milner, J.-C. (1978). De La Syntaxe à l'interprétation. Quantités, insultes, exclamations, Paris: Seuil.

Milner, J.-C. (1989). Introduction à une science du langage, Paris: Seuil.

Ouhalla, J. (1994). Transformational Grammar, London: Edward Arnold.

Pollock, J.Y. (1989). Verb movement, UG and the structure of IP', Linguistic Inquiry 20: 365-424. 
Rizzi, L. (1986). On the status of subjects clitics in Romance, in: R. Jaeggli \& C. Silva-Corvalan, (éds), Studies in Romance Linguistics, Dordrecht: Foris, 391-419.

Rouveret, A. (1987). Présentation \& Postscript, in: Chomsky N., La Nouvelle Syntaxe, Paris: Seuil.

Safir, K. (1986). Subject clitics and the Nom-Drop Parameter, in: Hagit Borer, (éd), Syntax and Semantics, 19, The Syntax of Pronominal Clitics, Orlando, Florida: Academic Press.

Sperber, D. \& Wilson, D. (1989). La Pertinence: communication et cognition, Paris: Minuit.

Tasmowski, L. \& D. Willems (1987).Les Phrases à première position actancielle vide, Travaux de linguistique, Gand, 13/14.

Vetters, C., (éd), (1993). Le Temps, de la phrase au texte, Lille: PUL.

Vetters, C. (1996). Temps, aspect et narration, Amsterdam: Rodopi.

Wagner, R.-L. (1939). Les Phrases hypothétique commençant par "si" dans la langue française des origines à la fin du XVIe siècle, Genève: Droz. 